\title{
Оптические переходы в кристаллах ZnSe и CdTe с участием $d$-зон катионов
}

\author{
(C) В.В. Соболев, Д.А. Перевощиков
}

Удмуртский государственный университет, 426034 Ижевск Россия

E-mail: sobolev@uni.udm.ru

(Получена 27 апреля 2017 г. Принята к печати 31 августа 2017 г.)

Спектры диэлектрических проницаемостей $\varepsilon_{1}(E)$ и $\varepsilon_{2}(E)$ кристаллов $\mathrm{ZnSe}$ и CdTe рассчитаны в области 10-25 эВ, используя их экспериментальные спектры отражения и интегральные соотношения Крамерса-Кронига. Они разложены на тринадцать и двенадцать отдельных полос переходов для $\mathrm{ZnSe}$ и CdTe соответственно, применяя усовершенствованный беспараметрический метод объединенных диаграмм Арганда. При этом определены их основные параметры: энергии максимумов и полуширин, силы осцилляторов. Значения сил осцилляторов находятся в интервалах $0.1-1.4(\mathrm{ZnSe})$ и $0.2-0.7$ (CdTe). Полученные полосы $\varepsilon_{2}(E)$ обусловлены межзонными и экситонными переходами с участием остовных $d$-зон катионов обоих кристаллов.

DOI: $10.21883 /$ FTP.2018.03.45613.8629

\section{1. Введение}

Халькогениды группы II-VI признаны одними из самых популярных полупроводниковых соединений при конструировании различных оптических приборов, гетероструктур и т.д. [1-4]. Поэтому зонная структуpa $\mathrm{ZnSe}$ и CdTe была подробно исследована различными экспериментальными методами. В частности, в [5-7] по фотоэмиссионным спектрам этих кристаллов установлено, что в отличие от популярных кристаллов $\mathrm{Si}, \mathrm{Ge}$ или группы III-V в области заполненных уровней между верхними $p$-состояниями и полуостовными $s$-состояниями расположены в относительно узкой области $d$-состояния катионов. Теоретические расчеты зон и оптических свойств $\mathrm{ZnSe}$ и $\mathrm{CdTe}$ проведены во многих работах [8-27]. В некоторых из них [8-10] использование метода псевдопотенциала из-за „упрощенности“ модели не позволило получить структуру $d$-зон. Более современные первопринципные методы расчета в приближении локальной плотности (LDA) или обобщенного градиентного приближения (GGA) позволяют получить $d$-зоны, но с большой погрешностью значения энергий $(\sim 2-3$ эВ) [11-13,28]. Применение различных усложнений и модификаций моделей расчетов [14-16,27] и использование различных подгоночных параметров $[17,18]$ в сравнении с предыдущими работами [11-13] выявили следующую качественную закономерность для селенидов и теллуридов: $d$-зоны катионов смещаются в область меньших значений энергий на $\sim 2-3$ эВ, но их дисперсия не меняется.

В [18,29-31] при температурах от жидкого азота до комнатной приведены экспериментальные данные о влиянии $d$-зон катионов на спектры отражения $(R(E))$ кристаллов ZnSe и CdTe в области от 10 до 25 эB: они содержат по три-четыре ярко выраженных (10-15 эВ) и более широких (15-25 эВ) максимумов и столько же ступенек (10-25 эВ). Теоретические расчеты оптических свойств (отражения, диэлектрической проницаемости и т.д.) для $\mathrm{ZnSe}$ и $\mathrm{CdTe}$ наиболее корректно проведены для области от 0 до $\sim 7$ эВ $[8,11,19,20]$. При бо́льших энергиях теоретические расчеты проведены в $[18,21,22]$. B [21] для CdTe определены парциальные вклады межзонных переходов с $d$-зон без учета спин-орбитального взаимодействия со множеством максимумов. В [18] для $\mathrm{ZnSe}$ также определены парциальные вклады межзонных переходов с $d$-зон, но с учетом спин-орбитального взаимодействия. При этом в $[18,22]$ установлено, что при $E>10$ эВ интенсивности теоретически рассчитанных спектров оптических функций отличаются от аналогичных экспериментальных значений в несколько раз.

Настоящая работа посвящена моделированию оптических переходов из $d$-зон кристаллов $\mathrm{ZnSe}$ и $\mathrm{CdTe}$ в области энергии 10-25 эВ и анализу полученных данных по дополнительным теоретическим расчетам зон и межзонных переходов.

\section{2. Теория и метод расчета}

Для определения реальной $\left(\varepsilon_{1}(E)\right)$ и мнимой $\left(\varepsilon_{2}(E)\right)$ частей диэлектрической проницаемости $\varepsilon(E)$ по известному спектру отражения широкое распространение получил метод на основе интегральных соотношений Крамерса-Кронига и аналитических формул связи между оптическими функциями. Данные методики нами подробно описаны в [26] и обсуждены в [32,33].

По спектру интегральной функции $\varepsilon_{2}(E)$ можно определить количество электронов, участвующих в переходах до заданной энергии $E_{0}$ :

$$
n_{\mathrm{eff}}\left(E_{0}\right)=A \frac{\Omega}{z} \int_{0}^{E_{0}} E \varepsilon_{2}(E) d E,
$$

где $A=0.462$ эВ $^{-2} \cdot$ нм $^{-3}-$ универсальная постоянная, $\Omega$ - объем элементарной ячейки (в нм$\left.{ }^{3}\right), z=4-$ число функциональных единиц в элементарной ячейке. 
Разложение спектров $\varepsilon_{2}(E)$ на элементарные компоненты и определение энергий их максимумов и полуширин было выполнено в рамках усовершенствованного беспараметрического метода объединенных диаграмм Арганда (ОДА). Беспараметрический метод ОДА основан на предположении, что элементарная компонента оптического перехода имеет форму симметричного лорентцевого осциллятора [32-34]:

$$
\begin{gathered}
\varepsilon_{1, i}(E)-1=\frac{\varepsilon_{2 \max , i} H_{i} E_{i}\left(E_{i}^{2}-E^{2}\right)}{\left(E_{i}^{2}-E^{2}\right)^{2}+H_{i}^{2} E^{2}}, \\
\varepsilon_{2, i}(E)=\frac{\varepsilon_{2 \max , i} H_{i}^{2} E_{i} E}{\left(E_{i}^{2}-E^{2}\right)^{2}+H_{i}^{2} E^{2}},
\end{gathered}
$$

где $E_{i}-$ энергия максимума, $H_{i}-$ полуширина и $\varepsilon_{2 \text { max }, i}-$ максимальное значение полосы $\varepsilon_{2, i}(E)$. Отличительная особенность такой изолированной полосы состоит в том, что ее диаграмма Арганда (зависимость $\left.\varepsilon_{2, i}=F\left(\varepsilon_{1, i}\right)\right)$ представляет идеальную окружность. Фундаментальная значимость этой окружности состоит в том, что ее параметры (диаметр, координаты ее центра) однозначно определяют параметры функций $\varepsilon_{1, i}$ и $\varepsilon_{2, i}: E_{i}, H_{i}$ и $\varepsilon_{2 \max , i}$. ОДА суммирует вклады множества окружностей отдельных полос, при этом вклад наиболее интенсивных проявляется структурно в виде большей части своей окружности. Достраивая проявляющиеся неполные окружности на кривой ОДА полными окружностями, можно в итоге получить все неизвестные параметры в уравнениях (2) и (3) для каждой выделенной полосы. Это позволяет шаг за шагом разложить весь спектр диэлектрической функции на отдельные компоненты без использования подгоночных параметров. Однако у этого метода есть недостаток: боковые хвосты лорентцевого осциллятора имеют физически необоснованную протяженность в широких областях энергии. Этому противоречит теоретически ожидаемое формирование полосы в весьма ограниченной области энергии. Несмотря на слабость, эти хвосты могут приводить к сильному занижению интенсивности слабых полос переходов, вплоть до их невозможности выделения. Естественно было усовершенствовать беспараметрический метод ОДА заменой слабых боковых хвостов касательными к контуру полос на уровне $\varepsilon_{2}$ max,i $/ 4$, что эквивалентно замене начальной формы кривой лорентциана на гауссиан.

Зная контур элементарной полосы функции $\varepsilon_{2}(E)$, можно, аналогично формуле (1), определить количество участвующих в переходах электронов в элементарной ячейке при формировании данной полосы (силу осциллятора):

$$
f_{i}=A \frac{\Omega}{z} \int_{a_{i}}^{b_{i}} E \varepsilon_{2, i}(E) d E,
$$

где $a_{i}$ и $b_{i}-$ начало и конец элементарной полосы.

Для многих кристаллов известны методы воспроизведения интегрального спектра набором ряда полос, используя 15-30 подгоночных параметров [31,34]. Существенное отличие примененной нами методики от известных многопараметрических моделей состоит в полном отсутствии подгоночных параметров и предварительной модели природы переходов.

Так как в нашей модели не заложена природа выделяемой полосы, она может быть обусловлена межзонными, экситонными, переходами или их комбинацией в случае малой энергии связи экситонов относительно полуширины полосы. Для определения природы возникновения выделенных полос мы будем опираться на модель межзонных переходов в рамках первопринципных методов расчета зон [35] и возможных метастабильных экситонов [26,27].

Расчеты зон и межзонных переходов проводились нами на основе теории функционала электронной плотности при использовании обменно-корреляционного потенциала GGA-PBESol [36], реализованной в пакете программ exciting [37]. Методы на основе GGAпотенциалов при больших достоинствах имеют три недостатка: 1) сильное занижение значения запрещенной зоны $[15-17,26], 2)$ некорректное описание интенсивности оптических переходов в верхние зоны проводимости $[18,22], 3)$ завышение положений остовных уровней $[15,17,27,28]$. Для решения первой проблемы уже стало нормой простое смещение зон проводимости на постоянное значение энергии $\delta_{\text {gap }}$ до согласования теоретического и экспериментального значений запрещенной зоны. Для устранения второго недостатка наши расчеты ограничим рассмотрением лишь парциальных вкладов межзонных переходов с $d$-уровней. Для существенного уменьшения третьей проблемы аналогично [28] введем смещение $\delta_{d}$ для интересующих нас остовных $d$-состояний. В результате общая поправка $\delta_{\mathrm{gap}+d}$ для межзонных переходов с участием остовных $d$-зон будет равна сумме $\delta_{\text {gap }+d}=\delta_{\text {gap }}+\delta_{d}$. В последующем для краткости интересующие нас межзонные переходы в зоны проводимости $C_{i}(i=1-6)$ будем обозначать $d \rightarrow C_{i}$ или $d(5) \rightarrow C_{i}$ и $d(3) \rightarrow C_{i}$, если надо выделить переходы из $d^{5 / 2}$ - и $d^{3 / 2}$-состояний соответственно.

На основе полученных зон можно также провести анализ по возможным экситонным переходам, которые сопровождают межзонные переходы при энергии $E_{e x}=E\left(d \rightarrow C_{i}\right)-E_{\text {bind. }}$. где $E_{e x}-$ энергия экситонного перехода, $E\left(d \rightarrow C_{i}\right)$ - энергия межзонного перехода, $E_{\text {bind }}$ - энергия связи электронно-дырочной пары.

\section{3. Результаты расчетов}

Для определения спектров $\varepsilon_{1}(E)$ и $\varepsilon_{2}(E) \mathrm{ZnSe}$ и $\mathrm{CdTe}$ (рис. 1) использовались экспериментальные спектры отражения в области 4-20 (100 K), 20-30 (300 K) [18] и 0-5 [29], 5-30 эВ $(300 \mathrm{~K})$ [30] соответственно. Энергии максимумов и ступенек $\varepsilon_{1}(E)$ и $\varepsilon_{2}(E)$ приведены в табл. 1 и в большинстве случаев смещены в область меныших значений энергии на $0.09-0.5$ эВ $\left(\varepsilon_{1}\right)$, $0.03-0.2$ эВ $\left(\varepsilon_{2}\right)$ и $0.1-0.6$ эВ $\left(\varepsilon_{1}\right), \quad 0.04-0.3$ эВ $\left(\varepsilon_{2}\right)$ 
Таблица 1. Энергии максимумов и ступенек интегральных спектров $\varepsilon_{1}(E), \varepsilon_{2}(E)$ (в эВ), рассчитанных на основе экспериментальных спектров отражения ZnSe и CdTe. Энергии ступенек приведены в скобках

\begin{tabular}{c|c|c|c|c|c}
\hline \multicolumn{3}{c|}{ ZnSe } & \multicolumn{3}{c}{ СdTe } \\
\hline $\begin{array}{c}\text { № } \\
\text { полосы }\end{array}$ & $\varepsilon_{1}$ & $\varepsilon_{2}$ & $\begin{array}{c}\text { № } \\
\text { полосы }\end{array}$ & $\varepsilon_{1}$ & $\varepsilon_{2}$ \\
\hline 1 & 11.31 & 11.37 & 1 & 12.04 & 12.13 \\
2 & $(11.8)$ & $(11.9)$ & 2 & 12.71 & $(12.9)$ \\
3 & $(12.2)$ & $(12.2)$ & 3 & $(13.1)$ & 13.25 \\
4 & 12.27 & 12.51 & 4 & $(13.5)$ & 13.65 \\
5 & 12.74 & 12.84 & 5 & 15.04 & 15.30 \\
6 & $(13.0)$ & $(13.1)$ & 6 & $(15.7)$ & $(15.8)$ \\
7 & 13.5 & $(13.8)$ & 7 & $(16.7)$ & $(16.8)$ \\
8 & 15.6 & 15.7 & 8 & 18.60 & 18.85 \\
9 & 16.7 & 16.8 & 9 & $(20.7)$ & 21.1 \\
10 & $(18.7)$ & $(18.7)$ & & & \\
11 & 20.2 & 20.6 & & & \\
12 & 22.8 & 23.4 & & &
\end{tabular}

для $\mathrm{ZnSe}$ и $\mathrm{CdTe}$ соответственно. Качественно структуры полученных спектров $\varepsilon_{2}(E)$ обоих кристаллов схожи и можно выделить следующие основные участки: 1) первая одиночная полоса при $\sim 11.37$ ( $\mathrm{ZnSe})$ и 12.13 эВ (CdTe), 2) интенсивная триплетная структура в интервалах $12.4-13.4$ э $(\mathrm{ZnSe})$ и $12.7-14.1$ эВ (CdTe), 3) область переходов от $\sim 14.8$ до $\sim 17.5$ эВ, 4) два широких максимума при $E>20 \ni \mathrm{B}(\mathrm{ZnSe})$ и $E>18$ эВ (CdTe). Такое сходство может говорить об одинаковой природе этих структур, но не обязательно. Дополнительно к рассмотренным выше кривая $\varepsilon_{2}(\mathrm{ZnSe})$ содержит дополнительные ступеньки малой интенсивности в области между первой одиночной полосой и интенсивной триплетной структурой, а также при $\sim 14$ и $\sim 19$ эВ. Функция $\varepsilon_{2}(\mathrm{CdTe})$ менее интенсивна,

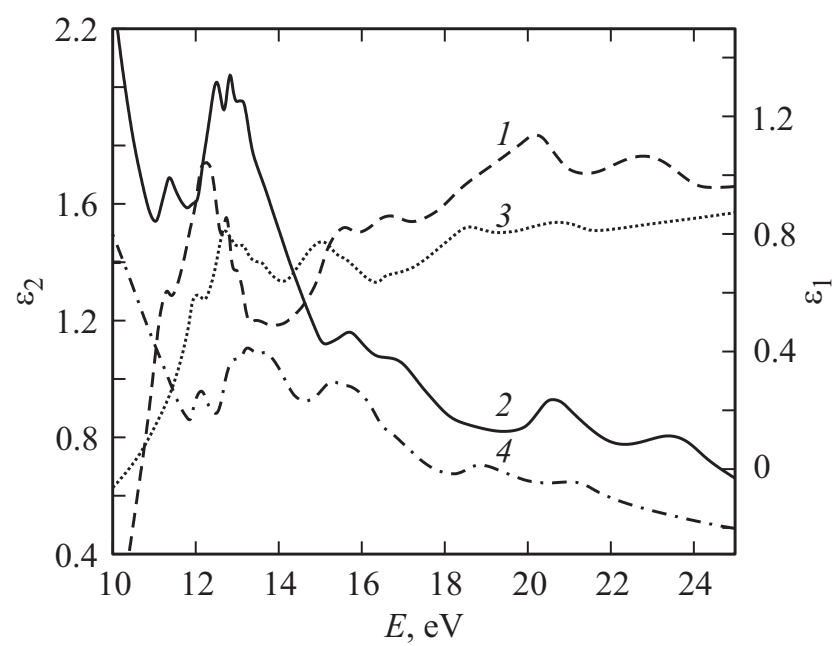

Рис. 1. Спектры экспериментально-расчетных кривых $\varepsilon_{1}(1,3)$ и $\varepsilon_{2}(2,4)$ для $\mathrm{ZnSe}(1,2)$ и $\mathrm{CdTe}(3,4)$ в области от 10 до 25 эВ. чем $\varepsilon_{2}(\mathrm{ZnSe})$, особенно в интервале от 10 до 15 эВ, где $\varepsilon_{2}(\mathrm{ZnSe})$ интенсивнее почти вдвое. При $E>15$ эВ разность $\varepsilon_{2}(\mathrm{ZnSe})-\varepsilon_{2}(\mathrm{CdTe}) \approx 0.2$. Эффективное общее количество электронов, участвующих в переходах в интервале $10-25$ эВ, для $\mathrm{ZnSe}$ меняется от $\sim 5.3$ до $\sim 11.1$, для СdТе - от $\sim 6.6$ до $\sim 12.8$, т.е. количество электронов элементарной ячейки, участвующих в переходах в заданной области энергии, у $\mathrm{ZnSe}$ и СdTe почти одинаково $(\sim 6)$, несмотря на то что $\varepsilon_{2}(\mathrm{ZnSe})>\varepsilon_{2}(\mathrm{CdTe})$ во всей рассматриваемой области. Связано это с тем, что объем элементарной ячейки $\mathrm{CdTe}$, входящий в уравнение (1), больше, чем у $\mathrm{ZnSe}$, в $\sim 1.5$ paза.

\subsection{ZnSe}

Используя усовершенствованный метод ОДА, кривая $\varepsilon_{2}$ была разложена во всей рассчитанной области. $\mathrm{B}$ настоящей работе данные для разложения приведены лишь для области от 10 до 25 эВ, в которой кривая $\varepsilon_{2}(E)$ была разложена на 13 полос оптических переходов (рис. 2). В табл. 2 приведены их энергии максимумов и полуширин, а также значения $\varepsilon_{2 \max , i}$ и определяемые по формуле (4) силы осцилляторов. При $E>14$ эВ влияние сплошного спектра межзонных переходов $d \rightarrow C_{i}$ не столь значительно, поэтому расположенные в этой области полосы имеют заметно меньшие значения полуширин $\left(H_{i} \approx 0.4-0.7\right.$ эВ) и силы осцилляторов $\left(f_{i} \approx 0.1-0.3\right)$, чем те, что расположены при бо́льших энергиях $\left(H_{i} \approx 1.1-4\right.$ эВ и $\left.f_{i} \approx 0.4-1.4\right)$.

Для определения природы возникающих полос переходов мы воспользовались первопринципными методами расчета зон и межзонных переходов. На вставке рис. 2 представлена зонная структура $\mathrm{ZnSe}$ вдоль осей высокой симметрии $\Lambda$ и $\Delta$ зоны Бриллюэна (за начало отсчета принято положение абсолютного минимума нижней зоны проводимости (НЗП)). Теоретически полученное значение запрещенной зоны $(1.04 \ni \mathrm{B})$ ожидаемо сильно занижено в сравнении с экспериментальным значением ( 2.75 эВ при $100 \mathrm{~K}[2])$, соответственно поправка $\delta_{\text {gap }}=1.7$ эВ. Другие теоретические расчеты зонной структуры $\mathrm{ZnSe}$ приведены в большом списке работ $[8-11,13,16-18,23,24]$, сильно отличающиеся между собой используемыми моделями и соответственно значениями запрещенных зон (в том числе в точках $L$ и $X$ зоны Бриллюэна). Поэтому для сравнения такого большого набора данных по зонам проводимости наиболее простым выходом является согласование всех этих результатов (в том числе наших) по положению НЗП в точке Г. Исходя из данного подхода можно сказать, что отличие наших значений $E_{g}^{(\Gamma-L)}$ и $E_{g}^{(\Gamma-X)}$ от большинства рассмотренных теоретических данных $[8-11,13,17,18]$ составляет $\sim 0.1$ эВ, с данными из [16] $\sim 0.2-0.3$ эВ. Различие более чем на 0.5 эВ выявлено результатами работ $[23,24]$. Поэтому с большой уверенностью можно говорить о корректности рассчитанных нами зон проводимости. В работе [38] 
Таблица 2. Основные параметры разложения $\varepsilon_{2}(E) \mathrm{ZnSe}$ энергия максимума $E_{i}$ (эВ), полуширина $H_{i}$ (эВ), $\varepsilon_{2}$ max, $i$ и сила осциллятора $f_{i}$

\begin{tabular}{r|c|c|c|c}
\hline$i$ & $E_{i}$ & $H_{i}$ & $\varepsilon_{2 \max , i}$ & $f_{i}$ \\
\hline 1 & 11.37 & 0.74 & 1.12 & 0.24 \\
2 & 11.92 & 0.64 & 0.88 & 0.17 \\
3 & 12.24 & 0.42 & 0.76 & 0.10 \\
4 & 12.51 & 0.39 & 1.23 & 0.15 \\
5 & 12.84 & 0.45 & 1.20 & 0.17 \\
6 & 13.13 & 0.41 & 0.94 & 0.13 \\
7 & 13.61 & 0.73 & 1.25 & 0.31 \\
8 & 14.4 & 1.1 & 0.97 & 0.39 \\
9 & 15.7 & 1.7 & 0.85 & 0.56 \\
10 & 16.9 & 1.8 & 0.67 & 0.50 \\
11 & 18.6 & 2.0 & 0.58 & 0.54 \\
12 & 20.6 & 2.2 & 0.64 & 0.72 \\
13 & 23.4 & 4.1 & 0.59 & 1.40
\end{tabular}

экспериментально проведено зондирование НЗП $\mathrm{ZnSe}$ вдоль направлений $\Lambda$ и $\Delta$ зоны Бриллюэна и определены значения запрещенных зон в точках $\Gamma, L$ и $X$. Проводя аналогичное согласование положения зон проводимости, получаем, что экспериментальные результаты для $E_{g}^{(\Gamma-L)}$ и $E_{g}^{(\Gamma-X)}$ отличны от наших лишь на $\sim 0.1$ эВ. Полученное нами значение спин-орбитального расщепления $d$-зон цинка $\Delta_{s o}(\mathrm{Zn} 3 d)=0.40$ эВ. К сожалению, прямых достоверных экспериментальных значений данной величины для $\mathrm{ZnSe}$ нет. Для расчета межзонных переходов положение $\mathrm{Zn} 3 d$-состояний примем на уровне среднего значения данных работ $[5,6](\sim 9.05$ эВ) и, как следствие, получаем значения поправок $\delta_{d}=2.6$ и $\delta_{\mathrm{gap}+d}=4.3$ эВ.

Выделенные полосы 1 и 2 возникают вследствие экситонных переходов в центре зоны Бриллюэна. Отношение количества электронов, участвующих при формировании

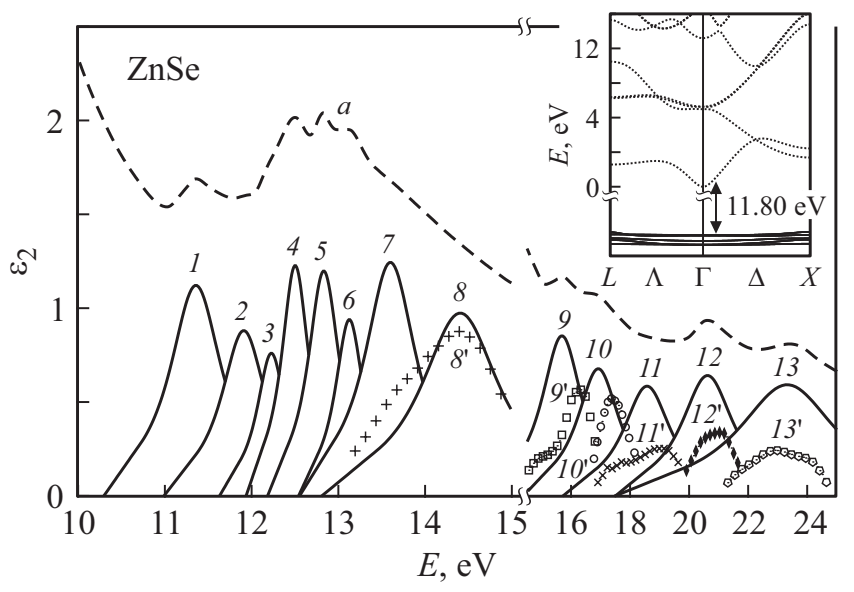

Рис. 2. Интегральный спектр кривой $\varepsilon_{2}(a)$, полосы ее разложения (1-13), а также зонная структура (вставка) и парциальные вклады межзонных переходов (в отн. ед.) ZnSe: $8^{\prime}-d \rightarrow C_{1}, 9^{\prime}-d \rightarrow C_{2}, 10^{\prime}-d \rightarrow C_{3}, 11^{\prime}-d \rightarrow C_{4}$, $12^{\prime}-d \rightarrow C_{5}, 13^{\prime}-d \rightarrow C_{6}$. этих полос, равно $f_{1} / f_{2} \approx 1.41$, что хорошо согласуется с теоретически ожидаемым значением 3/2. Следующие пять выделенных полос (3-7) полностью или частично формируются благодаря экситонным переходам в точках $L$ и $X$. К сожалению, более конкретную природу возникновения установить нельзя без дополнительной информации. В случае полосы 7 вклад также дает ступенька в межзонных переходах $d \rightarrow C_{1}$ при энергии $E_{s h}=13.7$ эВ. Следующая полоса соответствует межзонным переходам $d \rightarrow C_{1}$ с энергией максимума при $E_{\max }=14.4$ эВ, что хорошо согласуется с теоретическими данными работы [18]. Сильное влияние сплошного спектра межзонных переходов с участием $d$-зон цинка не позволило разрешить на интегральной кривой $\varepsilon_{2}(E)$ полосы 7 и 8. Энергии максимумов полос 9 и 10 смещены относительно соответствующих им максимумов межзонных переходов $d \rightarrow C_{2}\left(E_{\max }=16.3\right.$ эВ $)$ и $d \rightarrow C_{3}\left(E_{\max }=17.4\right.$ эВ $)$ на $\sim 0.5-0.6$ эВ. Проведенное сравнение с различными теоретическими расчетами зон с большой вероятностью позволяет предположить, что причиной сильного несоответствия положений максимумов полос 9 и 10 с максимумами межзонных переходов $d \rightarrow C_{2}$ и $d \rightarrow C_{3}$ является не погрешность в расчетах зон, а существование метастабильных экситонов, соответствующих максимумам этих межзонных переходов, с $E_{\text {bind }} \approx 0.5-0.6$ эВ. Полосы $11-13$ сформированы переходами из $d$-зон цинка в зоны проводимости $C_{4}, C_{5}$ и $C_{6}$, а также началом переходов из $s$-состояний селена, что также хорошо согласуется с данными работы [18]. Большие значения полуширин последних трех полос $\left(H_{i} \approx 2-4\right.$ эВ) вполне обоснованы, поскольку соответствующие им полосы межзонных переходов $d \rightarrow\left(C_{4}-C_{6}\right)$ также имеют большие значения полуширин.

\subsection{CdTe}

Как и в случае с ZnSe, усовершенствованным методом ОДА кривая $\varepsilon_{2}(\mathrm{CdTe})$ была разложена во всей рассчитанной области, но данные приведены лишь для области переходов от 10 до 25 эВ. Получено 12 полос оптических переходов (рис. 3). В табл. 3 приведены их основные параметры: $E_{i}, H_{i}, \varepsilon_{2 \max , i}$ и $f_{i}$. В отличие от $\mathrm{ZnSe}$ значения полуширин полос CdTe меняются заметно в меньшем диапазоне энергий от $\sim 0.6$ (полоса 2 ) до $\sim 1.7$ эВ (полоса 12 ), а диапазон значений сил осцилляторов $(\sim 0.2-0.7)$ меньше, чем у $\mathrm{ZnSe}$, почти в 2.5 раза. При $E>15$ эВ значение полуширин равно $\sim 0.7$ эВ, при бо́льших энергиях $-\sim 1.1-1.7$ эВ. Это различие также связано с влиянием сплошного спектра переходов $d \rightarrow C_{i}$.

Для определения природы полученных полос переходов мы использовали теоретические расчеты, аналогичные случаю $\mathrm{ZnSe}$. На вставке рис. 3 представлена зонная структура СdТе вдоль осей высокой симметрии $\Lambda$ и $\Delta$ зоны Бриллюэна (за начало отсчета принято положение абсолютного минимума НЗП). Теоретически рассчитанное значение запрещенной зоны $(0.38$ эВ) 
Таблица 3. Основные параметры разложения $\varepsilon_{2}(E)$ CdTe: энергия максимума $E_{i}$ (эВ), полуширина $H_{i}(э \mathrm{~B}), \varepsilon_{2}$ max, $i$ и сила осциллятора $f_{i}$

\begin{tabular}{c|c|c|c|c}
\hline$i$ & $E_{i}$ & $H_{i}$ & $\varepsilon_{2 \max , i}$ & $f_{i}$ \\
\hline 1 & 12.17 & 0.76 & 0.73 & 0.25 \\
2 & 12.85 & 0.61 & 0.63 & 0.18 \\
3 & 13.30 & 0.76 & 0.72 & 0.27 \\
4 & 13.85 & 0.68 & 0.68 & 0.23 \\
5 & 14.4 & 0.81 & 0.53 & 0.23 \\
6 & 15.22 & 1.1 & 0.74 & 0.44 \\
7 & 16.0 & 1.1 & 0.59 & 0.39 \\
8 & 16.9 & 1.2 & 0.51 & 0.37 \\
9 & 17.7 & 1.2 & 0.35 & 0.27 \\
10 & 18.8 & 1.4 & 0.53 & 0.52 \\
11 & 19.9 & 1.5 & 0.32 & 0.36 \\
12 & 21.1 & 1.7 & 0.51 & 0.66
\end{tabular}

меньше экспериментального ( 1.475 эВ при $300 \mathrm{~K}[2])$ на $\delta_{\text {gap }}=1.10$ эВ. Экспериментальных данных по величине запрещенных зон $E_{g}^{(\Gamma-L)}$ и $E_{g}^{(\Gamma-X)}$ для $\mathrm{CdTe}$ нами не было найдено, поэтому сравнение мы будем проводить лишь с данными теоретических работ [8$10,13,14,16,17,23-25]$. Аналогично корректируя положение всех зон по значению НЗП, в точке Г находим, что данные работ $[8-10,13,14,16,24,25]$ в точках $L$ и $X$ расходятся с нашими не более чем на $\sim 0.3$ эВ (из них $[8,14,24,25]$ - не более чем на $\sim 0.1$ эВ), т.е. расчеты зон можно считать корректными. Полученное спин-орбитальное расщепление $d$-зон составило $\Delta_{\text {so }}(\mathrm{Cd} 4 d)=0.70$ эВ, что хорошо согласуется с экспериментальными данными $0.63-0.83$ эВ $[5,7]$. Поправка

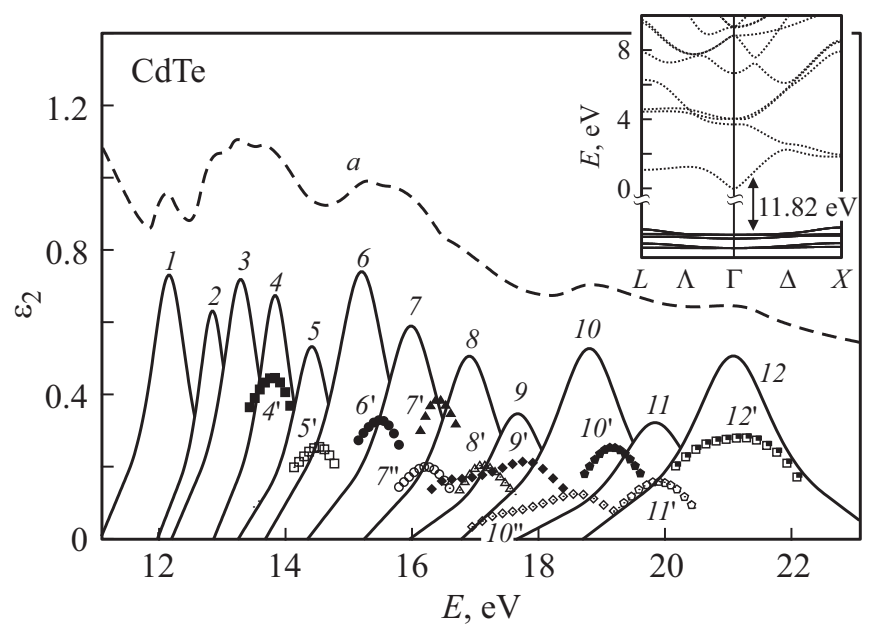

Pис. 3. Интегральный спектр кривой $\varepsilon_{2}(a)$, полосы ее разложения (1-12), а также зонная структура (вставка) и парциальные вклады межзонных переходов (в отн. ед.) CdTе: $4^{\prime}-d(5) \rightarrow C_{1}, 5^{\prime}-d(3) \rightarrow C_{1}, 6^{\prime}-d(5) \rightarrow C_{2}, 7^{\prime}-$ $d(5) \rightarrow C_{3}, 7^{\prime \prime}-d(3) \rightarrow C_{2}, 8^{\prime}-d(3) \rightarrow C_{3}, 9^{\prime}-d(5) \rightarrow C_{4}$, $10^{\prime}-d(5) \rightarrow C_{5}, \quad 10^{\prime \prime}-d(3) \rightarrow C_{4}, \quad 11^{\prime}-d(3) \rightarrow C_{5}$, $12^{\prime}-d \rightarrow C_{6}$. для межзонных переходов $d \rightarrow C_{i}(i=1-6)$ составила $\delta_{\mathrm{gap}+d}=3.14$ эВ. Подобные расчеты оптических переходов с $d$-состояний кадмия проведены в [21] без учета спин-орбитального взаимодействия. Энергии максимумов получены при 13.85, 15.8, 16.6 и 19.6 эВ. Их аналоги в нашей работе получены при $13.9\left(d \rightarrow C_{1}\right)$, $15.6\left(d \rightarrow C_{2}\right), \quad 16.7 \quad\left(d \rightarrow C_{3}\right) \quad$ и $\quad 19.4$ эВ $\quad\left(d \rightarrow C_{5}\right)$ соответственно, т.е. отличаются от предыдущих на $\sim 0.1-0.2$ эВ.

Теоретический анализ показывает, что первая одиночная полоса $\varepsilon_{2}(\mathrm{CdTe})$ в отличие от схожего максимума $\mathrm{ZnSe}$ связана с экситонными переходами в точке $L$ (из $d^{5 / 2}$-зон), а не Г. Полоса 2 также соответствует экситонным переходам в точке $L$ (из $d^{5 / 2}$-зон), полосы $3,4-$ в точке $X$. Также в случае полос 3 и 4 заметную роль начинает играть сплошной спектр межзонных переходов $d(5) \rightarrow C_{1}\left(E_{\max }=13.8\right.$ эВ). Отношение количества электронов, участвующих в формировании полос 1 и 2 , равно $\sim 1.4$, что близко к теоретически ожидаемому значению 3/2. Таким образом, интенсивные триплетные структуры в $\varepsilon_{2}(\mathrm{ZnSe})$ и $\varepsilon_{2}(\mathrm{CdTe})$ лишь качественно схожи по природе своего формирования (экситонные переходы в точках $L$ и $X$ ), при детальном же рассмотрении проявляются существенные различия. Пятая полоса формируется максимумом в межзонных переходах $d(3) \rightarrow C_{1}\left(E_{\max }=14.5\right.$ эВ $)$. Максимумы шестой и седьмой выделенных полос смещены в область меньших значений энергий на $\sim 0.4-0.5$ эВ относительно соответствующих им максимумов в межзонных переходах $d \rightarrow C_{2}\left(E_{\max }=15.6\right.$ эВ $)$ и $d \rightarrow C_{3}\left(E_{\max }=16.5\right.$ эВ $)$. Из рассмотренного выше сравнения зон можно с большой уверенностью предположить, что причиной этого являются экситоны перед максимумами в межзонных переходах $d \rightarrow C_{2}$ и $d \rightarrow C_{3}$ с $E_{\text {bind }} \approx 0.4-0.5$ эВ. Также частично на формирование полосы 7 оказывают влияние межзонные переходы $d(3) \rightarrow C_{2}\left(E_{\max }=16.2\right.$ эВ $)$. Восьмая выделенная полоса переходов соответствует межзонным переходам $d(3) \rightarrow C_{3} \quad\left(E_{\max }=17.1\right.$ эВ $)$ и $d(5) \rightarrow C_{4}\left(E_{\max }=16.7\right.$ эВ). При сравнении $\varepsilon_{2} \mathrm{ZnSe}$ и $\mathrm{CdTe}$ было отмечено их большое сходство в интервале энергий 14.8-17.5 эВ. Теоретический анализ показал, что у обоих этих кристаллов в данной области энергий формирование происходит за счет оптических переходов из $d$-состояний катионов во вторую и третью НЗП. Существенное отличие заключается в том, что спинорбитальное расщепление $d$-зон кадмия намного больше, чем у цинка, и поэтому для СdTе при разложении получено три полосы переходов (6,7 и 8), а не две (9 и 10). Полосы 9-12 сформированы межзонными переходами с $d$-состояний кадмия в зоны проводимости $C_{4}$, $C_{5}$ и $C_{6}$, т. е. последние два широких максимума $\mathrm{CdTe}$ совпадают по природе формирования с аналогичными двумя максимумами $\mathrm{ZnSe}$. Рассмотренные межзонные переходы $d(5) \rightarrow C_{i}$ и $d(3) \rightarrow C_{i}(i=1-6)$ СdТе имеют большие значения полуширин полос переходов, но не такую же значительную, как в ZnSe. Это обусловлено большей шириной зон проводимости $\mathrm{ZnSe}$, особенно при $E>6$ эВ (см. зоны на рис. 2 и 3 ). 
В работе [31] экспериментальная кривая отражения $\mathrm{CdTe}$ для $300 \mathrm{~K}$ в интервале $11-20$ эВ воспроизведена шестью лорентцевыми осцилляторами при 18 подгоночных параметрах. В отличие от наших результатов энергии их максимумов были заранее согласованы с энергиями соответствующих структур спектра $R(E)$, а полуширины и интенсивности выбирались путем минимизации получающейся разности между интегральной кривой отражения и суммой полос воспроизведения. Но энергии максимумов спектров отражения лишь приблизительно равны энергии максимумов соответствующих переходов и, как получено нами выше, эти отклонения примерно составляют $\sim 0.04-0.3$ эВ. Кроме того, в [31] предположена слишком малая интенсивность второго выделенного осциллятора, несмотря на то что на интегральной кривой отражения соответствующий ей максимум выражен почти как остальные максимумы.

\section{4. Заключение}

В настоящей работе рассчитаны диэлектрические функции кристаллов $\mathrm{ZnSe}$ и CdTe в области переходов с $d$-уровней катионов в интервале энергий от 10 до 25 эВ. Установлено, что в элементарной ячейке каждого кристалла при их формировании участвуют $\sim 6$ электронов с остовных уровней. Спектры $\varepsilon_{2}(E)$ разложены на 13 $(\mathrm{ZnSe})$ и $12(\mathrm{CdTe})$ полос переходов, определены энергии их максимумов и полуширин, силы осцилляторов. Значения сил осцилляторов изменяются в интервалах $f_{i}(\mathrm{ZnSe}) \approx 0.1-1.4$ и $f_{i}(\mathrm{CdTe}) \approx 0.2-0.7$. Теоретически рассчитаны остовные $d$-зоны обоих соединений и межзонные переходы из них в шесть нижних зон проводимости с учетом поправки величин запрещенных зон и положений $d$-состояний $\left(\delta_{\mathrm{gap}+d}=4.3(\mathrm{ZnSe})\right.$ и 3.14 эВ (CdTe)). Предложено существование экситонных переходов в точках $\Gamma, L$ и $X$ зоны Бриллюэна, а также связанных с максимумами в межзонных переходах с $E_{\text {bind }} \approx 0.4-0.6$ эВ.

\section{Список литературы}

[1] J.-H. Yang, W.-J. Yin, J.-S. Park, J. Ma, S.-H. Wei. Semicond. Sci. Technol., 31, 093002 (2016).

[2] В.В. Соболев, В.Вал. Соболев. Электронная структура твердых тел в области фундаментального края поглощения. Том II. Кристалль группы II-VI (Ижевск, УдГУ, 2012).

[3] R. Inoue, T. Abe, T. Fujimoto, N. Ikadatsu, K. Tanaka, Sh. Uchida, A. Tazue, H. Kasada, K. Ando, K. Ichino. Appl. Phys. Express, 8, 022101 (2015).

[4] Н. Морозова, Д. Мидерос, Н. Данилевич. Кислород в оптике соединений II-VI в свете теории антипересекающихся зон (Saarbrucken, Lap Lambert Academic Publishing, 2013).

[5] L. Ley, R.A. Pollak, F.R. McFeely, S.P. Kowalczyk, D.A. Shirley. Phys. Rev. B, 9, 900 (1974).

[6] C.J. Vesely, R.L. Hengehold, D.W. Langer. Phys. Rev. B, 5, 2296 (1972).
[7] N.J. Shevchik, J. Tejeda, M. Cardona, D.W. Langer. Phys. Status Solidi B, 59, 87 (1973).

[8] J.R. Chelikowsky, M.L. Cohen. Phys. Rev. B, 14, 556 (1976).

[9] O. Zakharov, A. Rubio, X. Blase, M.L. Cohen, S.G. Louie. Phys. Rev. B, 50, 10780 (1994).

[10] S.Zh. Karazhanov, L.C. Lew Yan Voon. ФТП, 39, 177 (2005).

[11] C.M.I. Okoye. Physica B, 337, 1 (2003).

[12] X.-Zh. Li, R. Gomez-Abal, H. Jiang, C. Ambrosch-Draxl, M. Scheffler. New J. Phys., 14, 023006 (2012).

[13] P. Gopal, M. Fornari, S. Curtarolo, L.A. Agapito, L.S.I. Liyanage, M.B. Nardelli. Phys. Rev. B, 91, 245202 (2015).

[14] E. Menendez-Proupin, A. Amezaga, C. Hernandez. Physica B, 452, 119 (2014).

[15] S. Sharma, J.K. Dewhurst, C. Ambrosch-Draxl. Phys. Rev. Lett., 95, 136402 (2005).

[16] A. Fleszar, W. Hanke. Phys. Rev. B, 71, 045207 (2005).

[17] J. Wang, Y. Zhang, L.-W. Wang. Phys. Rev. B, 92, 045211 (2015).

[18] R. Markowski, M. Piacentini, D. Debowska, M. ZimnalStarnawska, F. Lama, N. Zema, A. Kisiel. J. Phys.: Condens. Matter, 6, 3207 (1994).

[19] F. Kootstra, P.L. de Boeij, J.G. Snijders. Phys. Rev. B, 62, 7071 (2000).

[20] M. Grüning, D. Sangalli, C. Attaccalite. Phys. Rev. B, 94, 035149 (2016).

[21] R. Markowski, M. Podgorny. J. Phys.: Condens. Matter, 4, 2505 (1992).

[22] C.S. Wang, B.M. Klein. Phys. Rev. B, 24, 3417 (1981).

[23] H.H. Gürel, Ö. Akinci, H. Ünlü. Superlat. Microstr., 51, 725 (2012).

[24] X. Chen, X. Hua, J. Hu, J.-M. Langlois, W.A. Goddard. Phys. Rev. B, 53, 1377 (1996).

[25] J. Anversa, P. Piquini, A. Fazzio, T.M. Schmidt. Phys. Rev. B, 90, 195311 (2014).

[26] В.В. Соболев. Оптические свойства и электронная структура неметаллов. Том I. Введение в теорию (М.-Ижевск, Институт компьютерных исследований, 2012).

[27] F. Bechstedt. Many-body approach to electronic excitations. Concepts and applications (Berlin, Springer-Verlag, 2015).

[28] В.В. Соболев, Д.А. Перевощиков. ФТП, 51, 1078 (2017).

[29] J.L. Freeouf. Phys. Rev. B, 7, 3810 (1973).

[30] A. Kisiel, M. Zimnal-Starnawska, F. Antonangeli, M. Piacentini, N. Zema. Il Nuovo Cimento, 8D, 436 (1986).

[31] M. Krause, H.-E. Gumlich, U. Becker. Phys. Rev. B, 11, 6336 (1988).

[32] В.В. Соболев. Оптические свойства и электронная структура моноуглеродных сред. Том І. Алмаз. Графит. Аморфный углерод (Ижевск, УдГУ, 2016).

[33] В.В. Соболев. Оптические свойства и электронная структура моноуглеродных сред. Том II. Фуллериты. Нанотрубки. Графен. Интеркалированный графит (Ижевск, УдГУ, 2016).

[34] В.В. Соболев. Оптические свойства и электронная структура неметаллов. Том II. Моделирование интегральных спектров элементарными полосами (М.-Ижевск, Институт компьютерных исследований, 2012).

[35] R.O. Jones. Rev. Mod. Phys., 87, 897 (2015). 
[36] J.P. Perdew, A. Ruzsinszky, G.I. Csonka, O.A. Vydrov, G.E. Scuseria, L.A. Constantin, X. Zhou, K. Burke. Phys. Rev. Lett., 100, 136406 (2008).

[37] A. Gulans, S. Kontur, Ch. Meisenbichler, D. Nabok, P. Pavone, S. Rigamonti, S. Sagmeister, U. Werner, C. Draxl. J. Phys.: Condens. Matter, 26, 363202 (2014).

[38] А.А. Ланин, А.М. Желтиков. Письма ЖЭТФ, 104, 475 (2016).

Редактор Г.А. Оганесян

\section{Optical band transitions of the ZnSe and CdTe crystals with including cation $d$-bands}

\section{V.V. Sobolev, D.A. Perevoshchikov}

Udmurt State University, 426034 Izhevsk, Russia

Abstract Spectra of $\mathrm{ZnSe}$ and CdTe dielectrical permittivity $\varepsilon_{1}(E)$ and $\varepsilon_{2}(E)$ were calculated in the range 10 to $25 \mathrm{eV}$, using their experimental reflectivity spectra and integral Kramers-Kronig interrelations. Further more they were decomposed into the 13 and 12 simple transition bands by means of improved without parametrical method of combined Argand diagram with also their main parameters: energies of maxima and halhwidths, and oscillator strengths. The magnitudes of oscillator strengths are in the intervals 0.1 to $1.4(\mathrm{ZnSe})$ and 0.2 to $0.7(\mathrm{CdTe})$. The obtained $\varepsilon_{2}(E)$ bands were caused by interband and exciton transitions from the $d$-core cation bands for both crystals. 